\title{
Cost Effectiveness of Interval Cytoreductive Surgery With Hyperthermic Intraperitoneal Chemotherapy in Stage III Ovarian Cancer on the Basis of a Randomized Phase III Trial
}

Simone N. Koole, MD ${ }^{1,2}$; Christiaan van Lieshout, MSc ${ }^{1,3}$; Willemien J. van Driel, PhD, MD ${ }^{1,2,4,5}$; Evi van Schagen, MSc ${ }^{6}$; Karolina Sikorska, PhD ${ }^{1}$; Jacobien M. Kieffer, PhD ${ }^{1}$; Jules H. Schagen van Leeuwen, MD, PhD; Henk W.R. Schreuder, MD, PhD ${ }^{8}$; Ralph H. Hermans, MD, PhD ${ }^{9}$; Ignace H. de Hingh, MD, PhD ${ }^{5,9}$; Jacobus van der Velden, PhD, MD ${ }^{2,10}$; Henriette J. Arts, MD, PhD ${ }^{11}$; Leon F.A.G. Massuger, MD, PhD ${ }^{12}$; Arend G. Aalbers, MD ${ }^{1}$; Victor J. Verwaal, MD, PhD ${ }^{13}$; Koen K. Van de Vijver, MD, PhD ${ }^{14}$; Neil K. Aaronson, $\mathrm{PhD}^{1}$; Harm van Tinteren, $\mathrm{PhD}^{1}$; Gabe S. Sonke, MD, PhD ${ }^{1,4}$; Wim H. van Harten, MD, PhD ${ }^{1,3}$; and Valesca P. Retèl, PhD ${ }^{1,3}$

ASSOCIATED CONTENT

Data Supplements

Author affiliations and support information (if applicable) appear at the end of this article.

Accepted on May 17, 2019 and published at jco.org on June 28, 2019: DOI https://doi. org/10.1200/JC0.19. 00594

Clinical trial information: NCT00426257; EudraCT number: 2006-003466-34.

PURPOSE In the randomized open-label phase III OVHIPEC trial, the addition of hyperthermic intraperitoneal chemotherapy (HIPEC) to interval cytoreductive surgery (CRS) improved recurrence-free and overall survival in patients with stage III ovarian cancer. We studied the cost effectiveness of the addition of HIPEC to interval CRS in patients with ovarian cancer.

PATIENTS AND METHODS We constructed a Markov health-state transition model to measure costs and clinical outcomes. Transition probabilities were derived from the OVHIPEC trial by fitting survival distributions. Incremental cost-effectiveness ratio (ICER), expressed as euros per quality-adjusted life-year (QALY), was calculated from a Dutch societal perspective, with a time horizon of 10 years. Univariable and probabilistic sensitivity analyses were conducted to evaluate the decision uncertainty.

RESULTS Total health care costs were $€ 70,046$ ( $95 \%$ credibility interval [Crl], $€ 64,016$ to $€ 76,661$ ) for interval CRS compared with €85,791 (95\% Crl, €78,766 to €93,935) for interval CRS plus HIPEC. The mean QALY in the interval CRS group was 2.12 ( $95 \% \mathrm{Crl}, 1.66$ to $2.64 \mathrm{QALYS}$ ) and 2.68 (95\% $\mathrm{Crl}, 2.11$ to $3.28 \mathrm{QALYS}$ ) in the interval CRS plus HIPEC group. The ICER amounted to $€ 28,299 /$ QLYY. In univariable sensitivity analysis, the utility of recurrence-free survival and the number of days in the hospital affected the calculated ICER most.

CONCLUSION On the basis of the trial data, treatment with interval CRS and HIPEC in patients with stage III ovarian cancer was accompanied by a substantial gain in QALYS. The ICER is below the willingness-to-pay threshold in the Netherlands, indicating interval CRS and HIPEC is cost effective for this patient population. These results lend additional support for reimbursing the costs of treating these patients with interval CRS and HIPEC in countries with comparable health care systems.

J Clin Oncol 37:2041-2050. ๑ 2019 by American Society of Clinical Oncology

\section{INTRODUCTION}

Epithelial ovarian cancer has the highest mortality of all gynecologic tumors in the Western world. Most patients are diagnosed with International Federation of Gynecology and Obstetrics stage III to IV disease. ${ }^{1}$ The 10-year survival of women with advanced-stage ovarian cancer is $10 \%$ to $15 \%$ and has not improve in the past 20 years. ${ }^{2,3}$ Despite treatment with maximal cytoreductive surgery (CRS) and platinum-based chemotherapy, approximately $70 \%$ of patients with advanced-stage disease relapse within 18 months. Given this high number of recurrences, new approaches are needed to improve outcomes for these patients.
Because the peritoneal surface is the primary site of recurrence in ovarian cancer, administering chemotherapy directly in the abdominal cavity maximizes drug exposure. Local hyperthermia may have a direct cytotoxic effect and may increase drug sensitivity of tumor cells. We previously showed improved recurrence-free survival (RFS) and overall survival (OS) when hyperthermic intraperitoneal chemotherapy (HIPEC) was added to interval CRS in the multicenter randomized phase III OVHIPEC trial in patients with stage III ovarian cancer. ${ }^{6}$

For wide implementation of this technique and adequate financial coverage, cost-effectiveness analysis (CEA) is essential. The objective of this study was to 
perform a CEA, calculating the incremental cost-effectiveness ratio (ICER) of treatment with interval CRS and HIPEC compared with interval CRS for patients with stage III ovarian cancer.

\section{PATIENTS AND METHODS}

\section{Patients and Treatment}

The patient data used in the model were extracted from the OVHIPEC trial. In this multicenter randomized phase III trial, 245 patients with stage III ovarian cancer who received three neo-adjuvant chemotherapy cycles with carboplatin (area under the curve, 5 to $6 \mathrm{mg}$ ) and paclitaxel $175 \mathrm{mg} / \mathrm{m}^{2}$ were randomly assigned to receive interval CRS with or without HIPEC using cisplatin $100 \mathrm{mg} / \mathrm{m}^{2}$. One hundred twenty-two patients underwent interval CRS and HIPEC, and 123 patients received interval CRS only. Data were available on the surgical procedure, administered chemotherapy, hospital stay, additive diagnostic tests, and complications. Postoperative complications were determined and classified using the Common Terminology Criteria for Adverse Events, version 4, and grouped into toxicity grade 1 to 2 and grade 3 to 5 .

\section{Utility Estimates}

Patients in the OVHIPEC trial completed health-related quality of life (HRQoL) questionnaires of the European Organization for Research and Treatment of Cancer. No significant between-group differences were observed over time for the Quality of Life Questionnaire (QLQ)-C30 summary score in the OVHIPEC trial ( $p$-values for linear and non-linear growth: $p>0.133$ ). ${ }^{3 a}$ The questionnaires in the OVHIPEC trial did not include preference-based questions to obtain utilities. Because of the lack of mapping algorithms for ovarian cancer to derive utility values from QLQ-C30 results, we derived utilities from Havrilesky et al, ${ }^{4}$ as was previously done for cost-effectiveness studies in ovarian cancer. These utilities were based on time trade-off tasks performed by healthy women, women with ovarian cancer, and women previously treated for ovarian cancer. Nearly all patients were diagnosed with International Federation of Gynecology and Obstetrics stage III to IV ovarian cancer. The utility for diseasefree survival of 0.83 reported by Havrilesky et $\mathrm{al}^{4}$ was similar to utilities reported by other trials in advanced ovarian cancer. For both treatment arms in the OVHIPEC trial, the utility for the disease-free health state from Havrilesky et $\mathrm{al}^{4}$ was modified for the percentage of patients with grade 1 to 2 and in grade 3 to 5 toxicities, and for the percentage of patients receiving a colostomy. ${ }^{5}$ The risk of an adverse event or a colostomy was derived from the OVHIPEC trial (Table 1).

\section{Costs Estimates}

Total treatment costs from diagnosis to recurrent disease were calculated based on the treatment schedule of the OVHIPEC trial (Table 2). Total costs included chemotherapy agents, diagnostic tests, outpatient visits, societal costs,

TABLE 1. Survival and Outcome Probabilities and Health-State Utilities

\begin{tabular}{|c|c|c|c|c|}
\hline Health State & Mean & SE & Distribution & First Author \\
\hline \multicolumn{5}{|c|}{ Probabilities interval CRS (standard treatment) } \\
\hline Toxicity grade $1-2$ & 0.71 & 0.04 & Beta & van Driel $^{6}$ \\
\hline Toxicity grade 3-5 & 0.25 & 0.04 & Beta & van Driel ${ }^{6}$ \\
\hline Colostomy & 0.11 & 0.03 & Beta & van Driel ${ }^{6}$ \\
\hline ICU stay, days & 0.85 & 0.25 & Gamma & van Driel ${ }^{6}$ \\
\hline Hospital ward stay, days & 8.4 & 0.39 & Gamma & van Driel ${ }^{6}$ \\
\hline \multicolumn{5}{|c|}{ Probabilities interval CRS and HIPEC (investigational treatment) } \\
\hline Toxicity grade $1-2$ & 0.71 & 0.04 & Beta & van Driel ${ }^{6}$ \\
\hline Toxicity grade $3-5$ & 0.27 & 0.04 & Beta & van Driel $^{6}$ \\
\hline Colostomy & 0.17 & 0.03 & Beta & van Driel ${ }^{6}$ \\
\hline ICU stay, days & 2.05 & 0.40 & Gamma & van Driel $^{6}$ \\
\hline Hospital ward stay, days & 11.00 & 1.11 & Gamma & van Driel $^{6}$ \\
\hline \multicolumn{5}{|c|}{ Health-state utilities for both treatment groups } \\
\hline Recurrence-free survival & 0.83 & 0.06 & Beta & Havrilesky $^{4}$ \\
\hline Toxicity grade $1-2$ & 0.60 & 0.08 & Beta & Havrilesky ${ }^{4}$ \\
\hline Toxicity grade 3-5 & 0.49 & 0.09 & Beta & Havrilesky ${ }^{4}$ \\
\hline Recurrent disease, toxicity grade $3-5$ & 0.47 & 0.09 & Beta & Havrilesky ${ }^{4}$ \\
\hline Stoma disutility & -0.11 & 0.05 & Beta & Smith $^{5}$ \\
\hline
\end{tabular}

NOTE. SEs belonging to the utilities were calculated using reported standard deviations and sample sizes. SEs for survival probabilities, complication rates, and hospitalization days were derived from the OVHIPEC trial.

Abbreviations: CRS, cytoreductive surgery; HIPEC, hyperthermic intraperitoneal chemotherapy; ICU, intensive care unit. 
TABLE 2. Treatment Costs per Patient

Cost, $€$

Unit Cost Units (No.)

Costs

SE

Subtotal

Distribution

First Author

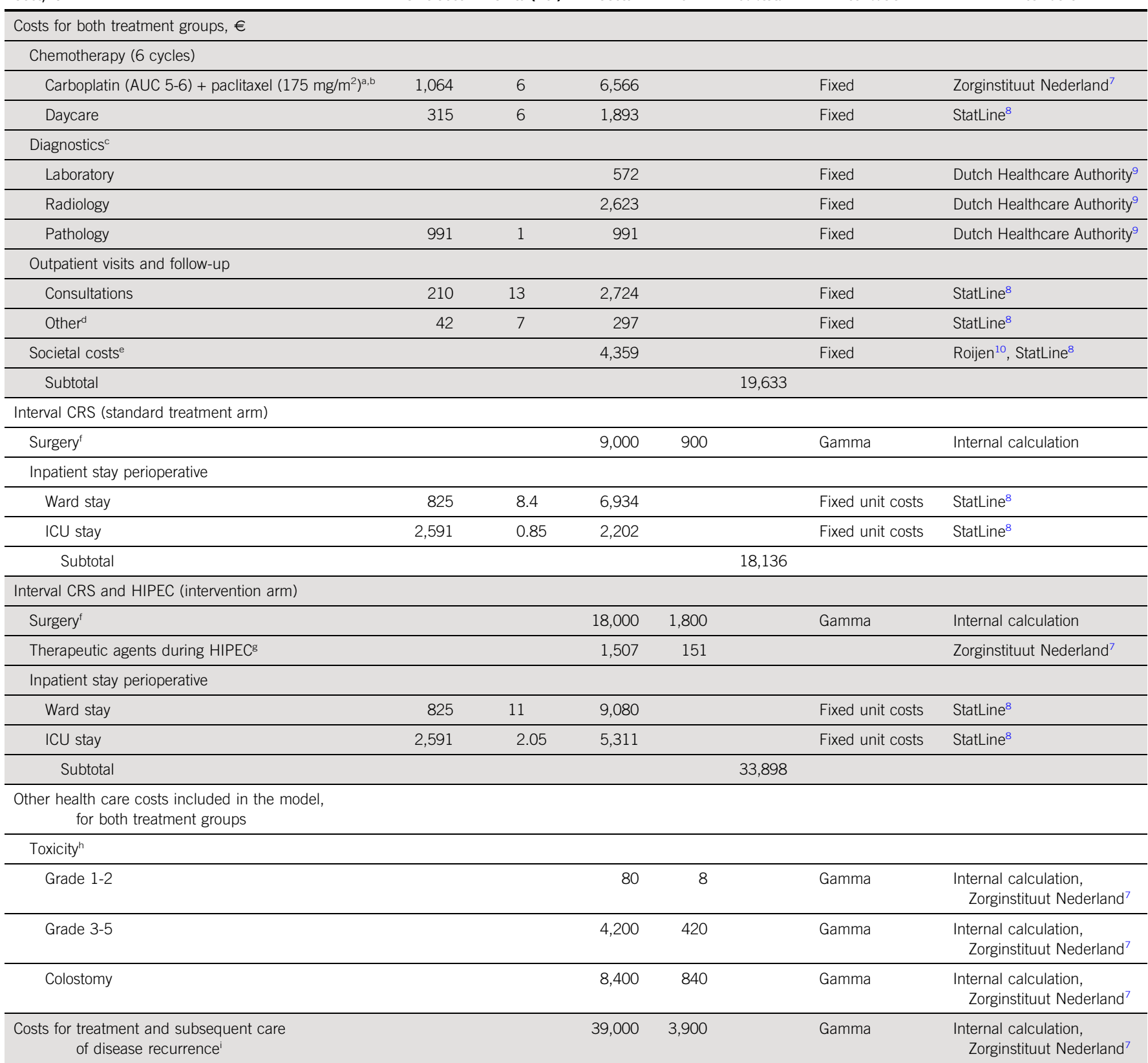

NOTE. Costs are expressed in 2017 euros. SEs for the costs were derived from internal cost calculations or estimated at a percentage of 10\%.

Abbreviations: AUC, area under the curve; CRS, cytoreductive surgery; HIPEC, hyperthermic intraperitoneal chemotherapy; ICU, intensive care unit.

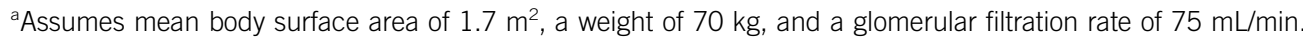

${ }^{\mathrm{b}}$ Includes materials and premedication (dexamethasone $8 \mathrm{mg}$, clemastine $2 \mathrm{mg}$, and ranitidine $150 \mathrm{mg}$ ).

${ }^{c}$ Consists of costs for multiple blood sample evaluations, computed tomography scans, ultrasound scans, $x$-rays, and the pathologic analyses of biopsies and resection specimens. Because costs for laboratory tests and for radiology modalities were grouped, units could not be given.

Including consultations by physiotherapists and dieticians.

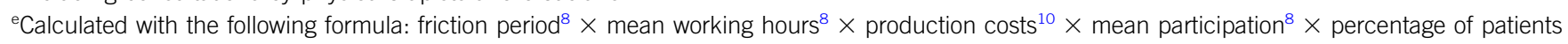

$<65$ years of age. ${ }^{6}$ Price per unit could not be expressed.

${ }^{\mathrm{f}}$ Consists of costs for anesthetic care and diagnostics; cytoreductive surgery, including abdominal uterus extirpation, removal of adnexa, omental tissue, and peritoneal disease; and an estimation of costs for surgical time, personnel costs, and, in the case of HIPEC, costs for disposables and use of the HIPEC perfusion system.

${ }^{8}$ Consists of costs for cisplatin and sodium thiosulfate.

${ }^{\text {h}}$ Costs for treatment of toxicities as reported previously in the OVHIPEC trial.

'Estimated costs for care at the event of recurrent disease, on the basis of treatment with standard chemotherapy. 
costs of surgery, costs of hospital admission, costs of admission to the intensive care unit (ICU), and estimated health care costs in case of toxicity. Costs of the most frequent grade 1 to 2 and 3 to 5 events in the OVHIPEC trial were calculated, taking into account costs for supportive drugs, readmission, and diagnostic tests. The number of hospitalization days, diagnostic tests, outpatient visits, and dosages and schedules for all administered regimens were based on the trial protocol, as described elsewhere. ${ }^{6}$ Costs were determined using multiple sources. Unit costs for inpatient hospital days, admission to the ICU, and costs for outpatient visits were derived from the Dutch National Health Care Institute (ZiNL) cost manual. ${ }^{10}$ Costs for chemotherapy and supportive drugs (ie, analgesia and anti-emetic drugs) were derived from the Dutch online database for therapeutic agents. ${ }^{7}$ Costs for imaging modalities, laboratory tests, and pathology review were determined using the maximum tariffs set by the Dutch Healthcare Authority (NZa) in 2017. For the estimation of surgery costs, we used the mean costs for CRS for a patient with ovarian cancer in 2017 in the Netherlands, as reported by the NZa. Costs for hospitalization days were reported separately. Costs for surgery with HIPEC were based on the prolonged duration of surgery, use of additional material and equipment, and personnel costs. Societal costs were calculated using data from the ZiNL cost manual and data provided by Statistics Netherlands. ${ }^{8,10}$ The period of production loss was calculated using the friction costs method for predicting the replacement time for a sick employee. ${ }^{10,11}$ The average labor costs per working day were determined using the weighted average labor costs of full-time and part-time employees in the Netherlands. The friction costs were assumed to be $80 \%$ of wage costs. Costs for colostomies were based on internal cost calculations and the ZiNL cost manual. Treatment of the most frequent Common Terminology Criteria for Adverse Events grade 1 to 2 and grade 3 to 5 toxicities were based on local protocols, and associated costs were derived from the Dutch online database for therapeutic agents. All costs were retrieved in 2017 euros or converted to 2017 euros by inflation rate using the Consumer Price Index.
Because of alternative treatment strategies for relapsed ovarian cancer, costs were calculated for three different treatment scenarios: (1) standard chemotherapy (base case); (2) carboplatin-gemcitabine-bevacizumab and maintenance bevacizumab for platinum-sensitive disease; and (3) maintenance poly (ADP-ribose) polymerase inhibitors for high-grade serous recurrent disease. Standard chemotherapy was considered to be treatment with carboplatin and paclitaxel and/or gemcitabine and/or doxorubicin.

The first scenario accounts for the lowest possible costs and is reported in the base case analyses to represent the most conservative strategy. Mean costs for disease recurrence for all three scenarios were based on real-time data from patients in the Netherlands Cancer Institute. Average costs for standard chemotherapy (first scenario) were calculated, taking into account the proportion of platinum-sensitive relapsed ovarian cancer in the OVHIPEC trial: 69\% had a platinum-sensitive relapse, and $31 \%$ had platinumresistant relapsed ovarian cancer. Associated costs for all scenarios were derived from the ZiNL cost manual, the $\mathrm{NZa}$, local costs, and the Dutch online database for therapeutic agents. ${ }^{7,9,10}$

\section{Model Description}

A Markov model was built to analyze the cost effectiveness of the addition of HIPEC to interval CRS. The model, constructed in Microsoft Excel, version 2010 (Microsoft, Redmond, WA), consisted of three mutually exclusive health states-RFS, disease recurrence, and death-with the corresponding utility for each health state (Fig 1). This study was performed from a societal perspective in the Netherlands. The duration of each cycle in the model was 3 months. A 10-year time horizon was chosen because of the life expectancy of the population and the reliability of the survival extrapolation.

Outcome probabilities. Survival data were extrapolated directly, using the exact patient-specific dates for disease recurrence and death from all patients included in the OVHIPEC trial. The following parametric survival models were fitted, according to the United Kingdom National

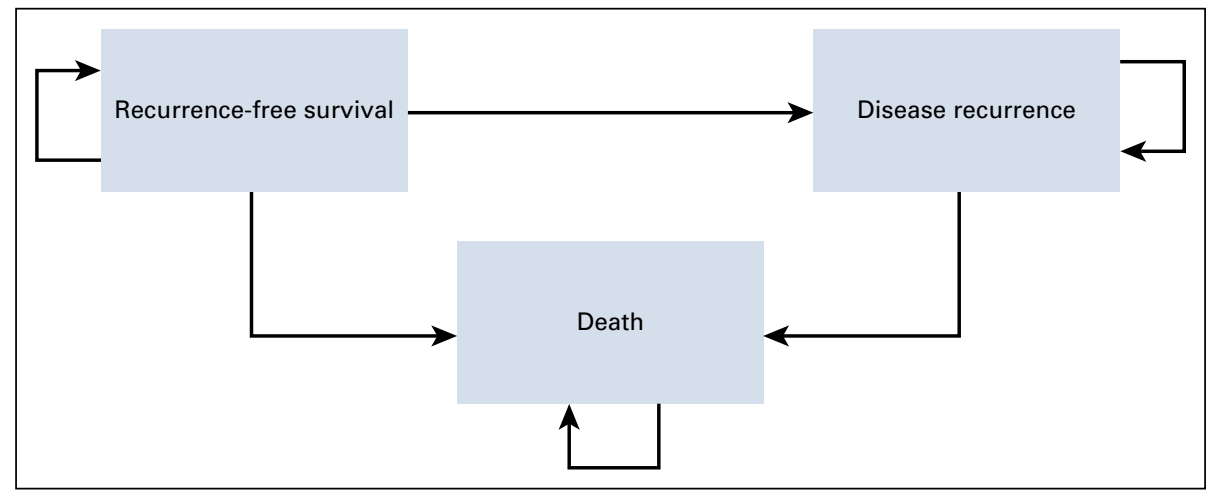

FIG 1. Schematic overview of the Markov model. 
Institute for Health and Care Excellence Decision Support Unit guidelines: the Gompertz, exponential, log-logistic, log-normal, and Weibull distributions. ${ }^{12}$ On the basis of the Akaike information criterion, visual comparison of the estimated parametric survival models and Kaplan-Meier plots of the data, and clinical knowledge of experts in our institute, the best model was selected (Data Supplement). The log-logistic distribution showed the best fit and was used for estimating survival and hazard rates of the observed 5-year follow-up period and for extrapolation beyond the observed time (Data Supplement). SEs of the estimated rates were obtained with 1,000 bootstrapped samples. Probabilities for RFS and OS $(p)$ per cycle $(t)$ were calculated using hazard rates $(r)$ for RFS and OS derived from the log-logistic model, using the following equation: $p=1-$ $\exp (-r t)$.

Markov model. To calculate the incremental mean costs and quality-adjusted life-years (QALY) difference between the two groups, we used bootstrapping with 1,000 replicas, representing women at 60 years of age, to derive an estimate and $95 \% \mathrm{Cl}$ for each treatment arm. The chosen age was based on the mean age of patients in the OVHIPEC trial. For each treatment arm, replicas entered the model at cycle 0 and remained in the same health state or transferred between health states at the end of each cycle, depending on probabilities for RFS or OS. Life-years (LYS) were calculated for both treatment arms. Subsequently, QALYs for both treatment arms were calculated based on probabilities for RFS, disease recurrence, grade 1 to 2 toxicity, grade 3 to 4 toxicity, and colostomies, with corresponding utilities and disutilities (Table 1). ${ }^{4,5}$ All cost and outcome data were discounted at a rate $4 \%$ and $1.5 \%$ per year, respectively, according to the Dutch guidelines. ${ }^{10,13}$ We assumed that all patients with recurrent disease, regardless of treatment arm, would be treated according to current treatment guidelines. The primary outcome of the model was the ICER. The ICER was calculated by dividing the mean incremental costs by the mean incremental QALY.

\section{Sensitivity Analyses}

We performed a series of sensitivity analyses to evaluate the robustness of the model and to address uncertainty in the estimation of variables. To determine which of the input parameters had the largest impact on the cost effectiveness of interval CRS and HIPEC, one-way sensitivity analyses were performed, showing the effect of a deviation of $20 \%$ of each individual model-input parameter on the ICER. Results were distributed in a tornado diagram.

A probabilistic sensitivity analysis (PSA) was performed using a Monte Carlo simulation, in which the simulation of 1,000 patients per treatment arm were repeated 1,000 times. Gamma distribution was used for cost parameters, and beta distribution was used for parameters bounded between 0 and 1 (Table 1). The corresponding ICERs for each of the iterations were illustrated in a cost-effectiveness plane. Cost-effectiveness acceptability curves were constructed to demonstrate the probability of cost effectiveness. In cost-effectiveness acceptability curves analysis, the willingness-to-pay (WTP) threshold for a certain health benefit is set against the probability of not breaching the threshold. In the Netherlands, the informal threshold for the WTP is $€ 80,000$ per QALY. ${ }^{14}$ To show the effect of increasing costs in the relapsed setting, a scenario analysis was performed using estimated costs of treatment with carboplatin-gemcitabine-bevacizumab and for treatment with maintenance poly (ADP-ribose) polymerase inhibitors for high-grade serous disease.

\section{RESULTS}

\section{Patients and Survival}

Demographic and baseline characteristics, treatment information, and patient outcome data of patients in the OVHIPEC trial are listed in the Data Supplement. The hazard ratio for RFS or death was $0.66(95 \% \mathrm{Cl}, 0.50$ to $0.87 ; P=.003)$. The hazard ratio for OS was $0.67(95 \% \mathrm{Cl}$, 0.48 to $0.94 ; P=.02){ }^{6}$

\section{Base Case Results}

The mean total health care costs of interval CRS and HIPEC were $€ 85,791$ (95\% credibility interval [Crl], $€ 78,766$ to $€ 93,935)$ compared with $€ 70,046$ (95\% Crl, $€ 64,016$ to $€ 76,661)$ for interval CRS, resulting in mean incremental costs of $€ 15,745$ (95\% Crl, $€ 5,829$ to $€ 25,927$ ) when adding HIPEC. Treatment with interval CRS and HIPEC led to a mean LY of $5.07(95 \% \mathrm{Crl}, 4.80$ to $5.34 \mathrm{LYs})$ compared with a mean of $4.07(95 \% \mathrm{Crl}, 3.83$ to $4.33 \mathrm{LYs})$ for patients treated with interval CRS only. This resulted in costs per LY gained of $€ 15,746$ when adding HIPEC. Adjusting for HRQoL, the mean QALYs in the interval CRS and HIPEC group was 2.68 (95\% Crl, 2.11 to 3.28 QALYS), whereas the mean QALYs in the interval CRS group was 2.12 (95\% Crl, 1.66 to 2.64 QALYS). This resulted in an ICER of $€ 28,299$ per QALY over the first 5 years for patients treated with interval CRS and HIPEC.

\section{Probabilistic Sensitivity Analysis}

The ICERs for the 1,000 samples in the PSA are shown in the scatter plot (Fig 2). Eighty-three percent of the points were under the $€ 80,000$-per-QALY level, and $92 \%$ of tested ICERs were in the northeastern quadrant. The costeffectiveness acceptability curve is shown in Figure 3 for varying values of WTP per QALY.

Results for the additional treatment scenarios after disease recurrence are listed in Table 3. With the increase of costs for the treatment of relapsed ovarian cancer, mean incremental costs for initial treatment with HIPEC decreases, resulting in a decrease of the calculated ICER.

\section{Univariable Sensitivity Analysis}

The results of the univariable sensitivity analysis are shown in the tornado diagram (Fig 4). The parameters with the 


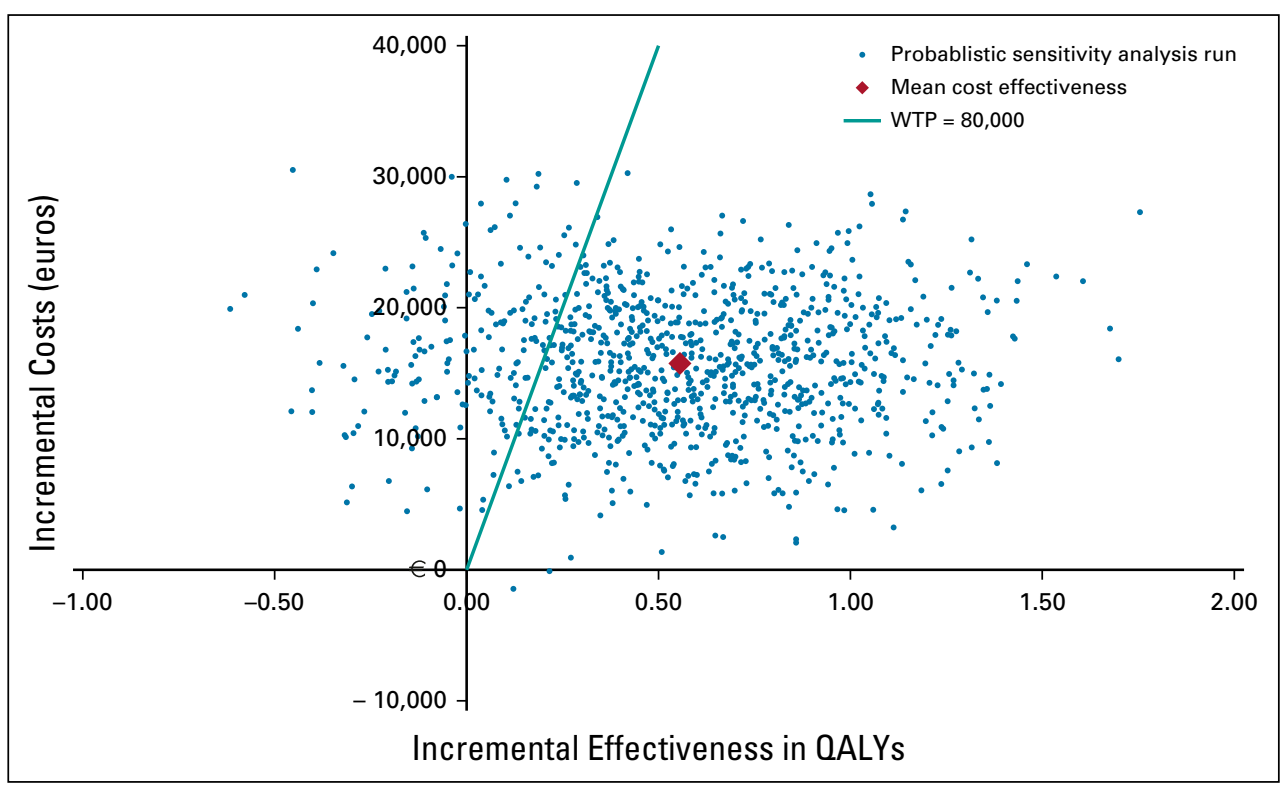

FIG 2. Cost-effectiveness plane for the incremental costs in euros compared with the incremental effectiveness in quality-adjusted life-years (QALYS), treatment with interval cytoreductive surgery, and hyperthermic intraperitoneal chemotherapy compared with treatment with interval cytoreductive surgery. Costs are expressed in 2017 euros $(€)$. The willingness-to-pay (WTP) threshold of $€ 80,000$ per QALY is pictured.

greatest influence on the ICER were the utility for RFS, the number of hospitalization days, and the utility for recurrent disease with grade 3 to 4 toxicity. Even with a broad variation in range for each parameter, the ICER remained below $€ 33,000$ per QALY.

\section{DISCUSSION}

We performed a CEA of treatment with interval CRS and HIPEC in patients with stage III ovarian cancer who were ineligible for primary CRS. Treatment with interval CRS and HIPEC resulted in a high incremental QALY benefit. In the randomized OVHIPEC trial, adding HIPEC to interval CRS prolonged OS by 11.8 months and increased costs by $€ 15,745$. For all scenarios tested in the univariable sensitivity analyses, the ICER remained below $€ 33,000$ per QALY. The PSA revealed that the probability of interval CRS and HIPEC being cost effective was $83 \%$ for the Dutch WTP threshold of $€ 80,000$ per QALY. On the basis of these data, treatment with interval CRS and HIPEC falls within the accepted values for cost-effective incremental costs of care in the Netherlands.

The WTP thresholds vary across countries and generally are based on the market value of goods and services of a country, expressed in per capita gross domestic product or the Commission on Macroeconomics and Health's corresponding estimate of the economic value of a QALY. ${ }^{15}$ Interventions that cost less than three times gross domestic product per capita are generally considered cost effective. ${ }^{16}$ The National Institute for Health and Care Excellence in England and Wales uses a range of $£ 20,000$ to $£ 30,000$ per QALY (€22,000 to $€ 33,000$ in 2014 euros). ${ }^{17}$ In the United States, ranges have been recommended but have

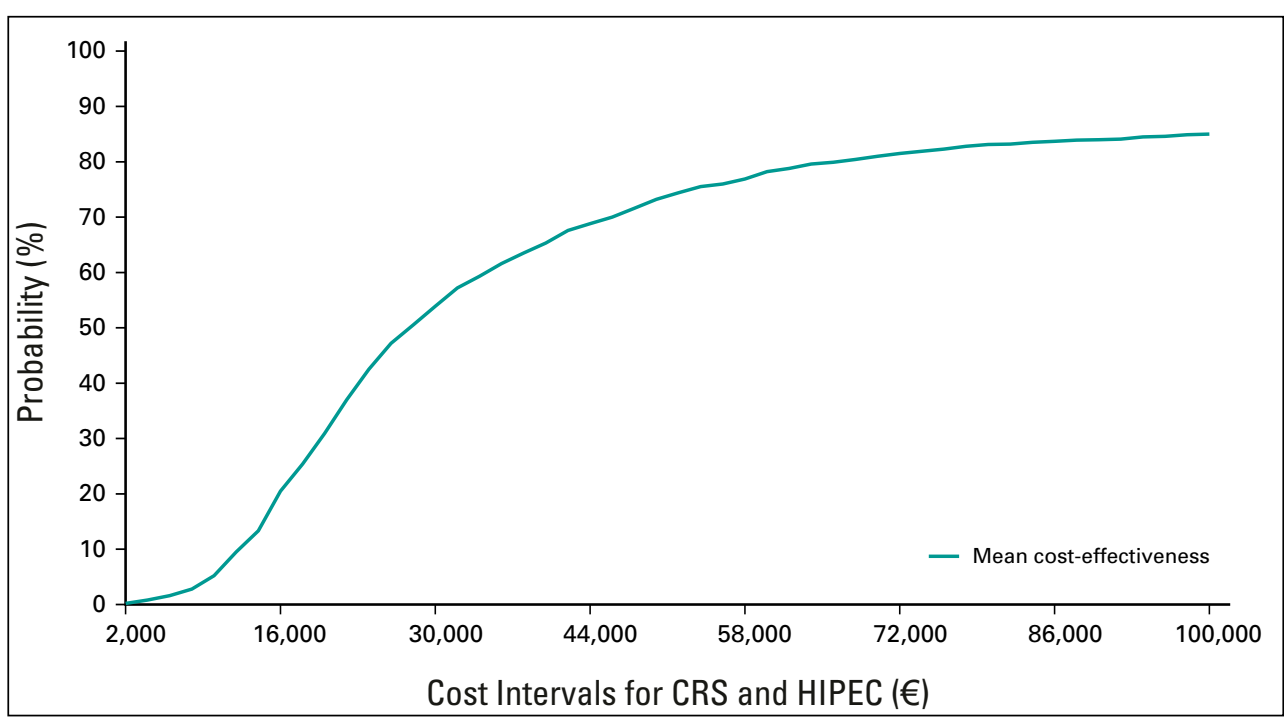

FIG 3. Cost-effectiveness acceptability curve showing the probability of interval cytoreductive surgery (CRS) and hyperthermic intraperitoneal chemotherapy (HIPEC) being cost effective given a certain willingness-to-pay threshold per quality-adjusted life-year in euros (€). Costs are expressed in 2017 euros. 
TABLE 3. Base Case Results for Initial Treatment With Interval CRS and HIPEC, and Scenario Analyses Calculated for Different Treatment Strategies for Relapsed Ovarian Cancer

Total Treatment Costs $(95 \%$ Crl)

Effectiveness

\begin{tabular}{|c|c|c|c|c|c|c|}
\hline Treatment & Mean Cost Estimation & Interval CRS & Interval CRS + HIPEC & Incremental Costs & Incremental QALY & ICER ( $€ /$ QALY) \\
\hline Standard chemotherapy & $39,000.00$ & $\begin{array}{c}70,046 \\
(64,016-76,661)\end{array}$ & $\begin{array}{c}85,791 \\
(78,766-93,935)\end{array}$ & $\begin{array}{c}15,745 \\
(5,829-25,927)\end{array}$ & 0.56 & 28,299 \\
\hline $\begin{array}{l}\text { Maintenance PARP } \\
\text { inhibitor for high-grade } \\
\text { serous recurrent disease }\end{array}$ & $100,000.00$ & $\begin{array}{c}116,435 \\
(101,604-132,347)\end{array}$ & $\begin{array}{c}131,202 \\
(116,790-147,831)\end{array}$ & $\begin{array}{c}14,766 \\
(8,090-37,208)\end{array}$ & 0.55 & 26,896 \\
\hline
\end{tabular}

NOTE. All costs are expressed in 2017 euros.

Abbreviations: $\mathrm{Crl}$, credibility interval; CRS, cytoreductive surgery; HIPEC, hyperthermic intraperitoneal chemotherapy; ICER, incremental costeffectiveness ratio; PARP, poly (ADP-ribose) polymerase; QALY, quality-adjusted life-year.

not been officially adopted and remain the subject of ongoing debate; both a range of $\$ 20,000$ to $\$ 100,000$ ( $€ 15,000$ to $€ 75,000$ ) and a threshold of $\$ 50,000$ $(€ 36,000)$ have been discussed. ${ }^{18,19}$ This CEA could support implementation and coverage decisions in countries with similar health care systems and expenditure ranges. Depending on the difference in treatment strategies and associated costs, the substantial gain in QALYs and the incremental costs demonstrated here might not translate into similar results in all countries.

Few observational and retrospective studies report the cost effectiveness of the HIPEC procedure. ${ }^{20-25}$ Study populations in these reports vary considerably, precluding their comparison. Our results provide the first evidence that

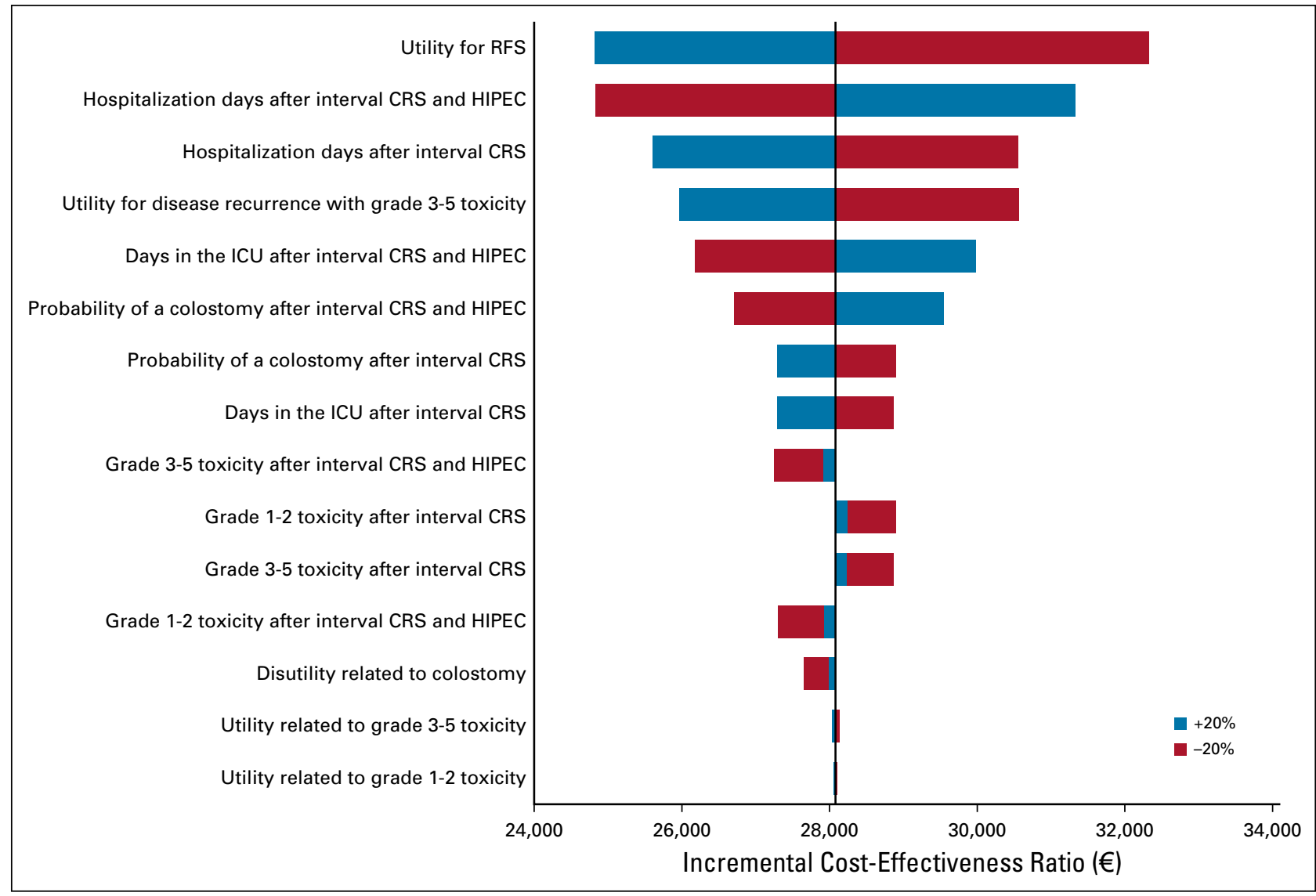

FIG 4. Tornado diagram showing one-way sensitivity analysis results. Bars indicate the effect of a $\pm 20 \%$ variance of a variable on the incremental cost-effectiveness ratio. Costs are expressed in 2017 euros $(€)$. Abbreviations: CRS, cytoreductive surgery; HIPEC, hyperthermic intraperitoneal chemotherapy; ICU, intensive care unit; QALY, quality-adjusted life-year; RFS, recurrence-free survival. 
HIPEC is cost effective, on the basis of data from a randomized controlled trial. The additional costs associated with HIPEC mainly result from the increased duration of surgery, capacity of the operation room, and prolonged hospitalization, including an additional day in the ICU (Table 1). These costs were estimated on the basis of internal costs and open sources in the Netherlands, but may vary across hospitals, across countries, and over time. Treatment might deviate from the standardized protocols used to design the model. In the OVHIPEC trial, HIPEC was performed in hospitals with expertise in performing HIPEC for patients with GI malignancies. Volumes and experience of the surgical team could influence the complication rate. The various cost elements related to the HIPEC procedure might vary among countries, although the procedure is well described and can be executed with a high degree of uniformity. Treatment with interval CRS and HIPEC results in fewer disease recurrences, resulting in a decline of the ICER when additional costs after disease recurrences increase (Table 3). ${ }^{6}$ Given this, treatment with interval CRS and HIPEC will be cost effective when combined with other costlier treatment strategies in the relapsed setting. ${ }^{26}$

Our study has some limitations. First, surgery costs, the number of diagnostic tests, the administered regimens and associated costs, and costs for toxicities and recurrences used in our analyses were based on assumptions. Second, the questionnaires used for the HRQoL analyses are not directly translatable to health-state utilities. Mapping algorithms might be used to derive utility values from QLQC30 results, but existing mapping algorithms have been tested and validated in different cancer populations without sufficient overlap with patients with ovarian cancer. ${ }^{27-29}$ In

\footnotetext{
AFFILIATIONS

${ }^{1}$ The Netherlands Cancer Institute, Amsterdam, the Netherlands ${ }^{2}$ Center for Gynecologic Oncology Amsterdam, Amsterdam, the Netherlands

${ }^{3}$ University of Twente, Enschede, the Netherlands

${ }^{4}$ The Dutch Gynecological Oncology Group, Utrecht, the Netherlands

${ }^{5}$ The Dutch Peritoneal Oncology Group, Eindhoven, the Netherlands

${ }^{6}$ Erasmus University Rotterdam, Rotterdam, the Netherlands

${ }^{7}$ St. Antonius Hospital, Nieuwegein, the Netherlands

${ }^{8}$ UMC Utrecht Cancer Center, Utrecht, the Netherlands

${ }^{9}$ Catharina Hospital, Eindhoven, the Netherlands

${ }^{10}$ Amsterdam University Medical Center, Amsterdam, the Netherlands

${ }^{11}$ University Medical Center Groningen, Groningen, the Netherlands

${ }^{12}$ Radboud University Medical Centre, Nijmegen, the Netherlands

${ }^{13}$ Aarhus University Hospital, Aarhus, Denmark

${ }^{14}$ University Hospital Ghent, Ghent, Belgium
}

\section{CORRESPONDING AUTHOR}

Valesca P. Retèl, PhD, Division of Psychosocial Research and Epidemiology, The Netherlands Cancer Institute, P.O. Box 90203, 1006 BE Amsterdam, the Netherlands; e-mail: v.retel@nki.nl. the absence of disease-specific validation, the mapping technique may lead to a bias of unknown magnitude and direction. Thus, we used previously reported utilities for each health state and for subsequent toxicities in both treatment arms. These utilities were based on a small sample, resulting in a relatively large degree of uncertainty in the PSA and a relatively low median cost-effectiveness probability (Figs 2 and 3). The univariable sensitivity analysis, however, shows that the ICER remains below $€ 33,000$ per QALY with a potential variance in utilities of $20 \%$. A more adequate estimation of the health-state utilities, preferably on the basis of prospective use of the EuroQoL-5-dimension questionnaire, would have resulted in less uncertainty and thereby a higher cost-effectiveness probability. ${ }^{30}$ Currently, an implementation study of OVHIPEC in the Netherlands is being conducted in which patient-specific utilities are being obtained prospectively. To validate these results, additional trials are warranted, collecting data on patient-specific utilities, costs, and outcomes, so that the level of uncertainty decreases. Nevertheless, the OVHIPEC trial provides unbiased estimates of the RFS and OS probabilities, the incidence of toxicities, and important treatment characteristics, such as hospital stay and duration of the surgical procedure.

In conclusion, this CEA demonstrated that, on the basis of the trial data, the addition of HIPEC to interval CRS results in a substantial gain in QALYs in patients with stage III ovarian cancer. The ICER compares favorably with the current WTP threshold in the Netherlands. This finding lends additional support for reimbursing treatment with interval CRS and HIPEC for these patients in countries with similar health care systems.

\section{EQUAL CONTRIBUTION}

S.N.K. and C.V.L. contributed equally to this work. W.H.v.H. and V.P.R. contributed equally to this work.

\section{SUPPORT}

The OVHIPEC clinical trial was funded by the Dutch Cancer Society.

AUTHORS' DISCLOSURES OF POTENTIAL CONFLICTS OF INTEREST AND DATA AVAILABILITY STATEMENT

Disclosures provided by the authors and data availability statement (if applicable) are available with this article at DOI https://doi.org/10.1200/ JCO.19.00594

\section{AUTHOR CONTRIBUTIONS}

Conception and design: Simone N. Koole, Christiaan van Lieshout, Willemien J. van Driel, Ralph H. Hermans, Arend G. Aalbers, Victor J. Verwaal, Neil K. Aaronson, Harm van Tinteren, Gabe S. Sonke, Wim H. van Harten, Valesca P. Retèl

Provision of study materials or patients: Willemien J. van Driel, Jules H. Schagen van Leeuwen, Ignace H. de Hingh, Jacobus van der Velden, Leon F.A.G. Massuger, Henk W.R. Schreuder, Ralph H. Hermans, 
Jacobus van der Velden, Henriette J. Arts, Arend G. Aalbers, Koen K. van de Vijver

Collection and assembly of data: Simone N. Koole, Christiaan van Lieshout, Evi van Schagen, Karolina Sikorska, Harm van Tinteren, Valesca P. Retèl

Data analysis and interpretation: Simone N. Koole, Christiaan van Lieshout, Willemien J. van Driel, Evi van Schagen, Jacobien M. Kieffer, Jules H. Schagen van Leeuwen, Henk W.R. Schreuder, Ralph H. Hermans, Ignace H. de Hingh, Koen K. Van de Vijver, Neil K. Aaronson, Harm van Tinteren, Gabe S. Sonke, Wim H. van Harten, Valesca P. Retèl Manuscript writing: All authors

Final approval of manuscript: All authors

Accountable for all aspects of the work: All authors

\section{ACKNOWLEDGMENT}

We thank K. Jozwiak for extrapolation of survival data. We thank the following individuals for contributing patients and for data collection for this study: R. van Ginkel (University Medical Centre Groningen, Groningen, the Netherlands), K. Havenga (University Medical Centre Groningen, Groningen, the Netherlands), A. Reyners (University Medical Centre Groningen, Groningen, the Netherlands), A. de Jong (Radboud University Medical Centre, Nijmegen, the Netherlands), P. Ottevanger (Radboud University Medical Centre, Nijmegen, the Netherlands), M. van der Ham (Radboud University Medical Centre, Nijmegen, the Netherlands), P. Witteveen (UMC Utrecht Cancer Centre, Utrecht, the Netherlands), M. Hermans (Sint Antonius Hospital, Nieuwegein, the Netherlands), M. Los (Sint Antonius Hospital, Nieuwegein, the Netherlands), M. Wiezer (Sint Antonius Hospital, Nieuwegein, the Netherlands), C. van Dam (Sint Antonius Hospital, Nieuwegein, the Netherlands), F. Kosterman (Academic Medical Centre, Amsterdam, the Netherlands), P. Tanis (Academic Medical Centre, Amsterdam, the Netherlands), A. Westermann (Academic Medical Centre, Amsterdam, the Netherlands), Y. Boomgaard (Catharina Hospital Eindhoven, Eindhoven, the Netherlands), D. van Loosdrecht (Catharina Hospital Eindhoven, Eindhoven, the Netherlands), R. Lembrechts (University Hospital Antwerp, Antwerpen, Belgium), P. van Dam (University Hospital Antwerp, Antwerpen, Belgium), and P. Vuylsteke (University Hospital Centre, University Catholique de Louvain Namur, Louvain, Belgium). We thank the following individuals for data collection and data management: A. Torres (The Netherlands Cancer Institute, Amsterdam, the Netherlands), J. Kant (The Netherlands Cancer Institute, Amsterdam, the Netherlands), L. Pronk (The Netherlands Cancer Institute, Amsterdam, the Netherlands), and D. Baars (The Netherlands Cancer Institute,

Amsterdam, the Netherlands). We thank The Netherlands

Comprehensive Cancer Organization (IKNL) for performing local data management.

\section{REFERENCES}

1. Heintz AP, Odicino F, Maisonneuve P, et al: Carcinoma of the ovary. FIGO 26th annual report on the results of treatment in gynecological cancer. Int J Gynaecol Obstet 95:S161-S192, 2006 (suppl 1)

2. Allemani C, Matsuda T, Di Carlo V, et al: Global surveillance of trends in cancer survival 2000-14 (CONCORD-3): Analysis of individual records for 37513025 patients diagnosed with one of 18 cancers from 322 population-based registries in 71 countries. Lancet 391:1023-1075, 2018

3. Timmermans M, Sonke GS, Van de Vijver KK, et al: No improvement in long-term survival for epithelial ovarian cancer patients: A population-based study between 1989 and 2014 in the Netherlands. Eur J Cancer 88:31-37, 2018

3a. Koole SN, Kieffer JM, Sikorska K, et al: Health-related quality of life after interval cytoreductive surgery with or without hyperthermic intraperitoneal chemotherapy (HIPEC) in patients with stage III ovarian cancer. Eur J Surg Oncol S0748-7983(19):30444-30445

4. Havrilesky LJ, Broadwater G, Davis DM, et al: Determination of quality of life-related utilities for health states relevant to ovarian cancer diagnosis and treatment. Gynecol Oncol 113:216-220, 2009

5. Smith DM, Sherriff RL, Damschroder L, et al: Misremembering colostomies? Former patients give lower utility ratings than do current patients. Health Psychol 25:688-695, 2006

6. van Driel WJ, Koole SN, Sikorska K, et al: Hyperthermic intraperitoneal chemotherapy in ovarian cancer. N Engl J Med 378:230-240, 2018

7. Zorginstituut Nederland: Zorginstituut Nederland, Price information. https://www.medicijnkosten.nl/

8. StatLine: Facts that matter, data portal. https://opendata.cbs.nl/statline/\#/CBS/en/navigatieScherm/thema

9. Dutch Healthcare Authority: Nederlandse Zorgautoriteit [NZa]. Pricelist healthcare products 2016 (Tarievenlijst DBC-zorgproducten en overige producten 2016). https://puc.overheid.nl/nza/

10. Hakkaart-van Roijen L, Van der Linden N, Bouwmans C, et al: Manual for Cost Analyses, Methods and Standard Prices for Economic Evaluations in Health Care. Diemen, the Netherlands, Zorginstituut Nederland (The National Health Care Institute), 2015

11. Garrison LP Jr, Mansley EC, Abbott TA III, et al: Good research practices for measuring drug costs in cost-effectiveness analyses: A societal perspective: The ISPOR Drug Cost Task Force report--part II. Value Health 13:8-13, 2010

12. Latimer NR: Survival analysis for economic evaluations alongside clinical trials--extrapolation with patient-level data: Inconsistencies, limitations, and a practical guide. Med Decis Making 33:743-754, 2013

13. Attema AE, Brouwer WBF, Claxton K: Discounting in economic evaluations. Pharmacoeconomics 36:745-758, 2018

14. Versteegh MM, Ramos IC, Buyukkaramikli NC, et al. Severity-Adjusted Probability of Being Cost Effective. Pharmacoeconomics. [Epub ahead of print on May 27, 2019]

15. Bertram MY, Lauer JA, De Joncheere K, et al: Cost-effectiveness thresholds: Pros and cons. Bull World Health Organ 94:925-930, 2016

16. Hutubessy R, Chisholm D, Edejer TT-T: Generalized cost-effectiveness analysis for national-level priority-setting in the health sector. Cost Eff Resour Alloc 1:8, 2003

17. National Institute for Health and Care Excellence: Guide to the methods of technology appraisal 2013. https://www.nice.org.uk/process/pmg9/resources/guideto-the-methods-of-technology-appraisal-2013-pdf-2007975843781

18. Bridges JF, Onukwugha E, Mullins CD: Healthcare rationing by proxy: Cost-effectiveness analysis and the misuse of the $\$ 50,000$ threshold in the US. Pharmacoeconomics 28:175-184, 2010

19. Vallejo-Torres L, García-Lorenzo B, Castilla I, et al: On the estimation of the cost-effectiveness threshold: Why, what, how? Value Health 19:558-566, 2016

20. Bonastre J, Chevalier J, Elias D, et al: Cost-effectiveness of intraperitoneal chemohyperthermia in the treatment of peritoneal carcinomatosis from colorectal cancer. Value Health 11:347-353, 2008

21. Baratti D, Scivales A, Balestra MR, et al: Cost analysis of the combined procedure of cytoreductive surgery and hyperthermic intraperitoneal chemotherapy (HIPEC). Eur J Surg Oncol 36:463-469, 2010 
22. Bryant J, Clegg AJ, Sidhu MK, et al: Clinical effectiveness and costs of the Sugarbaker procedure for the treatment of pseudomyxoma peritonei. Health Technol Assess 8:iii, 1-54, 2004

23. Chua TC, Martin S, Saxena A, et al: Evaluation of the cost-effectiveness of cytoreductive surgery and hyperthermic intraperitoneal chemotherapy (peritonectomy) at the St George Hospital peritoneal surface malignancy program. Ann Surg 251:323-329, 2010

24. Hultman B, Lundkvist J, Glimelius B, et al: Costs and clinical outcome of neoadjuvant systemic chemotherapy followed by cytoreductive surgery and hyperthermic intraperitoneal chemotherapy in peritoneal carcinomatosis from gastric cancer. Acta Oncol 51:112-121, 2012

25. Tentes AA, Pallas N, Korakianitis $\mathrm{O}$, et al: The cost of cytoreductive surgery and perioperative intraperitoneal chemotherapy in the treatment of peritoneal malignancy in one Greek institute. J BUON 17:776-780, 2012

26. Pujade-Lauraine E, Ledermann JA, Selle F, et al: Olaparib tablets as maintenance therapy in patients with platinum-sensitive, relapsed ovarian cancer and a BRCA1/2 mutation (SOLO2/ENGOT-Ov21): A double-blind, randomised, placebo-controlled, phase 3 trial. Lancet Oncol 18:1274-1284, 2017

27. Dakin H, Abel L, Burns R, et al: Review and critical appraisal of studies mapping from quality of life or clinical measures to EQ-5D: An online database and application of the MAPS statement. Health Qual Life Outcomes 16:31, 2018

28. Doble B, Lorgelly P: Mapping the EORTC QLQ-C30 onto the EQ-5D-3L: Assessing the external validity of existing mapping algorithms. Qual Life Res 25: 891-911, 2016

29. Woodcock F, Doble B: Mapping the EORTC-QLQ-C30 to the EQ-5D-3L: An assessment of existing and newly developed algorithms. Med Decis Making 38: 954-967, 2018

30. M Versteegh M, M Vermeulen K, MAA Evers S, et al: Dutch tariff for the five-level version of EQ-5D. Value Health 19:343-352, 2016

\section{ASCO Membership Delivers What You Need}

ASCO is unique in that we are the only oncology organization that serves members from all subspecialties and professional roles. We are also a global community of members from over 150 countries that takes an inclusive approach to the pursuit of quality cancer care and progress.

ASCO membership provides the support, resources, and solutions for all your professional needs:

- Stay on the cutting edge of scientific research and advances

- Streamline your pursuit of continuous learning

- Access evidence-based and data-driven quality resources

- Obtain insight into best practices for cancer care teams

- Connect and exchange views with oncology experts

To learn more about the value of ASCO membership, visit asco.org/membership

Not an ASCO member? Join today at join.asco.org 
Cost Effectiveness of Interval Cytoreductive Surgery With Hyperthermic Intraperitoneal Chemotherapy in Stage III Ovarian Cancer on the Basis of a Randomized Phase III Trial

The following represents disclosure information provided by authors of this manuscript. All relationships are considered compensated. Relationships are self-held unless noted. I = Immediate Family Member, Inst = My Institution. Relationships may not relate to the subject matter of this manuscript. For more information about ASCO's conflict of interest policy, please refer to www.asco.org/rwc or ascopubs.org/jco/site/ifc.

Ignace $\mathrm{H}$. de Hingh

Research Funding: Roche (Inst)

Gabe S. Sonke

Consulting or Advisory Role: Novartis (Inst)

Research Funding: Roche (Inst), AstraZeneca (Inst), Novartis (Inst), Merck Sharp \& Dohme (Inst)
Wim H. Van Harten

Research Funding: Agendia BV (Inst), Novartis (Inst)

Valesca P. Retèl

Research Funding: Agendia BV (Inst)

No other potential conflicts of interest were reported. 